\title{
El pensamiento multiplicativo de niños del pueblo indígena shipibo-konibo de Ucayali: una perspectiva piagetiana ${ }^{1}$
}

\author{
Jorge Villalba Garcés ${ }^{2}$ \\ ORCID: 0000-0002-2843-9854 \\ Susana Frisancho Hidalgo ${ }^{2}$ \\ ORCID: 0000-0002-5517-7597
}

\section{Resumen}

El presente estudio tuvo como objetivo identificar y describir los niveles de pensamiento multiplicativo de un grupo de niños de dos comunidades indígenas pertenecientes al pueblo shipibo-konibo de la región Ucayali, en la Amazonía del Perú. Participaron catorce estudiantes de nivel primario, de entre 7 y 12 años, tanto niñas como niños, que fueron evaluados utilizando el método clínico-crítico de Jean Piaget, con una tarea de multiplicación que contó con material manipulativo (peces de madera en tres tamaños diferentes y cuentas que representaban su alimento). Las respuestas de los niños frente a la tarea propuesta ponen en evidencia seis niveles de desarrollo del pensamiento multiplicativo, que son consistentes con los hallazgos de otras investigaciones y muestran la universalidad de las estructuras lógico-matemáticas. Los resultados indican también que los niños tienen dificultades para resolver la tarea de multiplicación, para tomar en cuenta las proporciones, y para dar argumentos que justifiquen sus respuestas. Los resultados se discuten enfatizando en la universalidad del proceso de construcción del conocimiento multiplicativo, los particulares culturales que pueden apoyar este proceso, y la necesidad de entender que, si bien la multiplicación se introduce en tercer grado, es una operación compleja incluso para niños de grados superiores.

\section{Palabras clave}

Educación matemática - Multiplicación - Shipibo-konibo.

1- Trabajo realizado en el marco el proyecto de Evaluación de competencias cognitivas y morales en niños adolescentes de comunidades Shipibo (Ucayali) y percepción sobre la educación en sus comunidades (Proyecto de Investigación 2014-0008) y financiado por la Pontificia Universidad Católica del Perú.

2- Pontificia Universidad Católica el Perú, Lima, Perú. Contactos: jvillalba@pucp.pe; sfrisan@pucp.edu.pe.

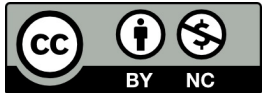




\section{Multiplicative thinking in Shipibo-konibo children from Ucayali, Peru: A Piagetian perspective}

\section{Abstract}

The objective of this study was to identify and describe levels of multiplicative thinking in a group of Shipibo-Konibo children from the Ucayali region, in the Peruvian Amazon rainforest. Fourteen elementary school students from two Shipibo-Konibo communities, boys and girls aged between 7 and 12 years old, participated in the study. They were evaluated using Jean Piaget's clinical-critical method, with a multiplication task that included manipulative materials (wooden fish in three different sizes, and plastic beads representing local food staples). The children's responses to the proposed task revealed six levels of development of multiplicative thinking, consistent with previous research findings and indicative of the universality of logical-mathematical structures. The study also found that children have difficulty to solve the multiplication task, take proportions into account, and offer arguments to justify their answers. These results are discussed with an emphasis on the universality of the process of multiplicative knowledge construction, the cultural specificities that can support this process, and the need to understand that multiplication, although it is introduced in the curricula at third grade, remains a complex operation even for children in higher grades.

\section{Keywords}

Mathematics education - Multiplication - Shipibo-konibo.

\section{Introducción}

La matemática es un producto cultural que se ha ido desarrollando a lo largo de la historia, cuyo dominio predice el nivel socioeconómico que alcanzará la persona (RITCHIE; BATES, 2013), y que en la actualidad resulta imprescindible para ejercer plenamente la ciudadanía (SKOVSMOSE, 1998; WOODROW, 1997). En la escuela primaria, los niños deben aprender las herramientas para el uso flexible de la matemática básica, incluyendo la operación de multiplicación. En el Perú, el Currículo Nacional (2016) propone introducir esta operación en 3er grado de primaria, año en el que ya se le pide al niño logros como la multiplicación de números naturales de hasta tres cifras.

Si bien muchos consideran que la multiplicación es simplemente una forma abreviada de adición, la primera es una operación mucho más compleja que la segunda, pues requiere mayores niveles de abstracción y relaciones de inclusión, las que muchas veces deben darse en simultáneo (PIAGET; INHELDER, 2007). Por ejemplo, tal como plantean Clark y Kamii (1996) desde el enfoque constructivista piagetiano, al sumar $3+3+3+3$ es necesario 
un solo nivel de abstracción, pues cada uno de los grupos de unidades de tres se encuentra en el mismo nivel: son unidades que se incluyen sucesivamente entre sí, a través de la siguiente operación: 3 unidades más otras 3 unidades $=6$ unidades; 6 unidades más 3 unidades $=9$ unidades; y 9 unidades más 3 unidades $=12$ unidades. Por el contrario, multiplicar 3x4 implica, primero, elaborar paquetes de 3 y establecer cada uno como una unidad independiente de las otras; segundo, en un siguiente nivel de abstracción, incluir una unidad de 3 en dos unidades de 3, dos unidades de 3 en tres unidades de 3 y tres unidades de 3 en cuatro unidades de 3, dando como resultado 12. Esta diferencia entre el pensamiento aditivo, que requiere un solo nivel de abstracción, y el pensamiento multiplicativo, que requiere dos niveles de abstracción, se grafica en la siguiente figura, tomada de Clark y Kamii (1996, p. 42):

Figura 1- Pensamiento aditivo y pensamiento multiplicativo

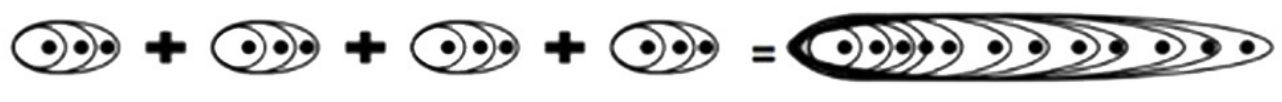

(a) Aditivo

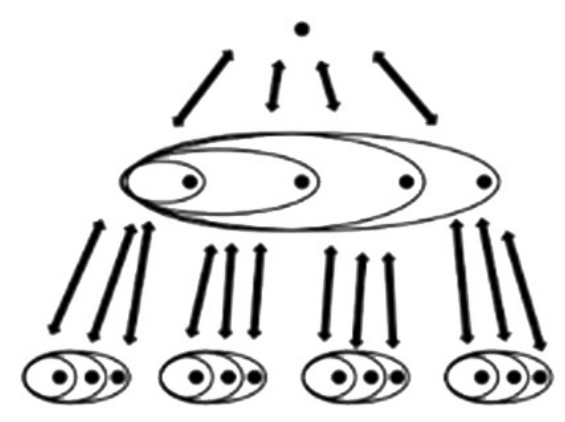

(b) Multiplicativo

Fuente: Adaptado de Clark y Kamii (1996).

Estudios muestran que la multiplicación es una operación compleja para los niños incluso de 9 años de edad (VAN DER VEN et al., 2015; SIEMON; BLEACKLY; NEAL, 2012; FERNANDEZ; LLINARES, 2010; SIEMON et al., 2006; CLARK; KAMII, 1996; KAMII, 1994). Bessa y Gonçalves da Costa (2016) verificaron la dificultad que tenían los estudiantes para comprender las relaciones multiplicativas, aun usando un juego de cartas que las facilitaba. Solo después de sucesivos procesos de toma de conciencia promovidos por los desafíos que proponía el juego, consiguieron percibir esas relaciones. Carraher, Carraher y Schliemann (1991) mostraron también que incluso niños que tenían un 
pensamiento matemático desarrollado presentaban dificultades con los códigos escritos de la matemática, y Cox (1974), en un estudio de dos años, ya había reconocido que el mayor número de errores aritméticos ocurrían en la multiplicación, cuyo algoritmo es tan complejo que muchos de los errores encontrados eran distintos y únicos para cada niño. En el Currículo Nacional peruano, la noción de doble, que ya requiere un pensamiento multiplicativo, se encuentra presente desde segundo grado de primaria (MINEDU, 2016), grado en el que se espera una media de edad de 7 años.

Los niños de pueblos originarios y de contextos culturales diversos, distintos al urbano occidental, suelen tener más conflictos y dificultades con el aprendizaje de las matemáticas (VERGARA-LOPE et al., 2017; SUBRAMANIAN, 2015; MEANEY; MCMURCHY-PILKINGTON; TRINICK, 2012; MOROMIZATO IZU, 2011; MEANEY, 2002). En el Perú, la Evaluación Censal de Estudiantes (ECE) (UMC, 2013, 2014) ha mostrado, a lo largo de los años, que los estudiantes de la Amazonía presentan los resultados más críticos en el aprendizaje de las matemáticas y no llegan a niveles satisfactorios de desempeño. En las pruebas ECE de los años 2015 y 2016 (UMC, 2015, 2016), si bien se aprecia una mejoría progresiva tanto a nivel nacional como en la región Ucayali, esta última sigue teniendo resultados muy por debajo de la media nacional. Asimismo, es posible encontrar diferencias entre el desempeño matemático de estudiantes cuya lengua materna es el castellano y el de estudiantes cuya lengua materna es una lengua originaria, a favor de los primeros, así como mejores desempeños en estudiantes de contextos urbanos, cuando se les compara con los de contexto rural.

Desde la etnomatemática, se ha enfatizado que las matemáticas son parte integral de cualquier grupo cultural, sea este alfabetizado o no (D'AMBROSIO, 2001), y se ha distinguido entre las matemáticas académicas, que son enseñadas y aprendidas en la escuela, y las prácticas matemáticas cotidianas de grupos culturalmente identificables, por ejemplo, al contar, clasificar, diseñar, o medir (ASHER, 2002; D’AMBROSIO, 1985, 2014). De este modo, se intenta explicar la diferencia en el desempeño escolar argumentando que no existen conocimientos matemáticos universales y que las evaluaciones escolares no respetan la matemática cultural (AROCA, 2016; FUENTES LEAL, 2014; PARRA; ORJUELA, 2014; MEANEY; EVANS, 2013; SANTOS; DONIZETI, 2011; JARAMILLO, 2011; SCHROEDER, 2005; BISHOP, 1995). Sin embargo, esta postura ha recibido críticas, tanto epistemológicas como pedagógicas, señalando por ejemplo que un conocimiento matemático no es correcto o verdadero por el solo hecho de ser una práctica cultural, y que el conocimiento escolar o académico es una forma de conocimiento distinta del conocimiento cotidiano (PAIS, 2011; HORSTHEMKE; SCHÄFER, 2007; ROWLANDS; CARSON, 2002).

Desde una perspectiva piagetiana, existen estudios que muestran que los procesos de construcción del conocimiento lógico matemático ocurren de modos muy similares aun en contextos culturales distintos (MISHRA, 2014; CARRETER0, 1981). Por ejemplo, la conservación, considerada como esencial para todas las actividades racionales (BRUNER; OLVER; GREENFIELD, 1966; PIAGET; SZEMINSKA, 1975), fue explorada por Nyiti (1976), quien investigó el desarrollo de la conservación de la sustancia, el peso y el volumen en un grupo de niños del grupo étnico Meru de Tanzania, tanto escolarizados como no escolarizados, y encontró que todos desarrollaron las capacidades de conservación en la 
misma secuencia invariable que los niños europeos de clase media con quienes se han realizado la mayoría de estudios piagetianos. Similares hallazgos fueron reportados por John y otros autores (1983) en Botswana, y por el mismo Piaget $(1971,1977)$ al indicar que, en relación a la secuencia de aparición de la conservación de substancia, peso y volumen, se encuentra la misma sucesión de estadios tanto en niños alfabetizados de la ciudad de Teherán como en niños analfabetos del campo y tanto en Irán como en Suiza. En todos los estudios, las escasas diferencias entre los grupos se dieron solo en la velocidad con la que se adquirieron los estadios. Villalba y Frisancho (2018) encontraron también similitudes entre los patrones de construcción de la suma y la resta en niños indígenas de una comunidad shipibo-konibo de la Amazonía del Perú, y los encontrados por Piaget y sus seguidores en sus estudios originales.

Ahora bien, Piaget (1974) ofreció cuatro razones por las cuales se afecta el desarrollo de un individuo: 1) factores biológicos, 2) factores de equilibración, 3) factores sociales y de coordinación interpersonal, que están presentes en todas las culturas, y 4) factores sociales, educativos y de trasmisión cultural que varían de cultura a cultura. De este modo, aunque las matemáticas son un conocimiento universal y hacer matemáticas es una capacidad humana asequible a todas las personas (SIEGLER; BRAITHWAITE, 2017; PIAGET, 1975, 1977; HALLPIKE, 1986), sí es cierto que el contexto sociocultural y las actividades propias de cada cultura son importantes y generan diferencias en el ritmo y el modo en que dicha capacidad se construye y expresa (WORTHINGTON; VAN OERS, 2016; NUNES, 1993; NUNES; CARRAHER; DIAS SCHLIEMANN, 1982). En este sentido, si bien la escuela tiene un rol fundamental en el desarrollo del pensamiento matemático de los niños, el conocimiento matemático no se construye únicamente dentro de la escuela sino también a partir de la participación en actividades culturales y prácticas como el tejido, el diseño de artesanías, el ordenamiento y clasificación de alimentos, o las transacciones de compra-venta o trueque que se realizan en la vida cotidiana (SCHROEDER, 2005; SCHLIEMANN et al., 1998; NUNES, 1993; NUNES; CARRAHER, DIAS SCHLIEMANN, 1982). Por ejemplo, al comparar a niñas Mayas Zinacantecas que sabian tejer con niños estadounidenses que no tejían se encontraron diferencias en las relaciones espaciales, que estaban más desarrolladas en las primeras. Igualmente, adolescentes sin escolaridad que tejían lograron construir diseños con muchos más detalles que los que hicieron estudiantes de college estadounidenses sin experiencia tejiendo, quienes solo produjeron representaciones simples, y carentes de detalles (GREENFIELD; CHILDS, 1977; GREENFIELD; MAYNARD; CHILDS, 2003). Ejemplos de otras actividades culturales tradicionales que promueven el pensamiento matemático son la confección de colchas con diseño geométrico en Norteamérica (WARES, 2006), la elaboración de canastas tradicionales en Mozambique o en el pueblo Ticuna de Brasil (COSTA; LUCENA, 2015; LAWRENCE, 2011), el origami (WARES, 2010, 2012, 2016; WARES; ELSTAK, 2013, 2017), o incluso el baile (VON RENESSE; EKCE, 2011).

Tomando en cuenta lo anterior, este estudio se planteó como objetivo identificar y describir las características del pensamiento multiplicativo de un grupo de niños de dos comunidades nativas shipibo-konibo de la región Ucayali, en la Amazonía peruana, región que, como se ha visto, presenta el más bajo rendimiento en las evaluaciones de desempeño matemático en el Perú. La idea de los investigadores fue constatar las 
capacidades multiplicativas de estos niños, utilizando un método cualitativo de evaluación y materiales manipulativos con los que ellos estuvieran más familiarizados.

\section{Método}

\section{Participantes}

Participaron catorce estudiantes de primaria, de entre 7 y 12 años, hombres y mujeres, de dos escuelas multigrado de educación intercultural bilingüe (EIB) de dos comunidades shipibo-konibo de la región Ucayali. El pueblo shipibo-konibo es el quinto grupo indígena del Perú en cuanto a población (PERÚ, 2017) y pertenece a la familia lingüística Pano. Los shipibo-konibo son un pueblo originario que tradicionalmente estuvo asentado en las cuencas del río Ucayali y sus afluentes. En la actualidad, sin embargo, existen comunidades shipibo-konibo en otras regiones del país, por ejemplo, en Madre de Dios, Loreto y Huánuco, e incluso en la ciudad de Lima donde han formado la única comunidad nativa de la capital.

La lengua materna de los participantes es el shipibo-konibo, aunque todos demostraron tener domino del español. La comunidad de Bethel se encuentra a 7 horas de distancia, en bote, de la ciudad de Pucallpa, mientras que la comunidad Bena Jema se ubica a 40 minutos de la misma ciudad, en el distrito de Yarinacocha. La selección de los participantes fue accidental, procurando contar con un rango de edad que permitiera observar diferencias en el desarrollo del pensamiento multiplicativo. Para acceder a los participantes de Bethel, se contó con el consentimiento de sus padres y de la comunidad, incluyendo al jefe de la misma y al profesor responsable de la escuela. Para acceder a los estudiantes de Bena Jema, se contó con el consentimiento de la directora de la escuela y del profesor a cargo del aula. A todos los niños se les explicó el objetivo de la evaluación y se les pidió su asentimiento. Las principales características de los participantes se presentan en la tabla siguiente:

Tabla 1- Principales características de los participantes del estudio

\begin{tabular}{cccccccc}
\hline Nombre & Edad & Sexo & Grado & Residencia \\
\hline Wil & 7 años & Masculino & Segundo & Bethel \\
\hline Luc & & 7 años & $\vdots$ & Femenino & Segundo & Bethel \\
\hline Les & $\vdots$ & 9 años & $\vdots$ & Femenino & $\vdots$ & Tercero & Bethel \\
\hline Man & $\vdots$ & 9 años & $\vdots$ & Masculino & $\vdots$ & Tercero & Bethel \\
\hline Dal & $\vdots$ & 11 años & $\vdots$ & Femenino & $\vdots$ & Quinto & Bethel \\
\hline Sha & $\vdots$ & 11 años & & Femenino & & Sexto & Bethel \\
\hline Maz & $\vdots$ & 8 años & $\vdots$ & Femenino & $\vdots$ & Tercero & Bena Jema \\
\hline Yal & $\vdots$ & 8 años & $\vdots$ & Masculino & $\vdots$ & Tercero & Bena Jema \\
\hline Key & $\vdots$ & 9 años & $\vdots$ & Femenino & $\vdots$ & Cuarto & Bena Jema \\
\hline Pat & $\vdots$ & 9 años & $\vdots$ & Femenino & $\vdots$ & Cuarto & Bena Jema \\
\hline Ale & $\vdots$ & 9 años & $\vdots$ & Masculino & $\vdots$ & Cuarto & Bena Jema \\
\hline Gis & $\vdots$ & 12 años & $\vdots$ & Femenino & $\vdots$ & Sexto & Bena Jema \\
\hline Ste & $\vdots$ & 12 años & $\vdots$ & Femenino & $\vdots$ & Sexto & Bena Jema \\
\hline Cri & $\vdots$ & 13 años & $\vdots$ & Masculino & $\vdots$ & Sexto & Bena Jema \\
\hline
\end{tabular}

Fuente: datos de la pesquisa. 


\section{Técnica de recolección de información}

A fin de identificar y explicar los procedimientos y la lógica usada por los niños al resolver los problemas, y no únicamente sus respuestas correctas o incorrectas, se utilizó la entrevista clínica-crítica de Jean Piaget, la que tiene como objetivo principal indagar en la lógica y el conocimiento que una persona ha construido sobre una tarea determinada. Este tipo de entrevista, que centra su interés en los niveles de organización sucesivos de la conducta, permite al evaluador formular contra-ejemplos o contra-sugestiones para profundizar en el razonamiento de la persona, y valoriza los cambios cualitativos por encima de los cuantitativos (PARRAT, 2016; DUCRET, 2004).

Con el método clínico-crítico el evaluador puede preparar un número específico de preguntas, pero durante la entrevista estas pueden multiplicarse en función de las respuestas que ofrece la persona (PARRAT, 2016). Además, el evaluador no solo llegará a conclusiones en torno a lo que el evaluado manifieste verbalmente sino también observando su manipulación del material (DELVAL, 2001).

\section{Tarea para la evaluación de la operación de multiplicación}

Se hizo uso de una tarea elaborada por Clark y Kamii (1996), basada en el método clínico crítico de Piaget. Se utilizaron tres peces de madera de 5 (pez A), 10 (pez B) y 15 (pez C) centímetros y cuentas de color azul que simulaban su alimento. En la siguiente figura se observan los materiales utilizados:

Figura 2- Materiales utilizados en la evaluación de pensamiento multiplicativo

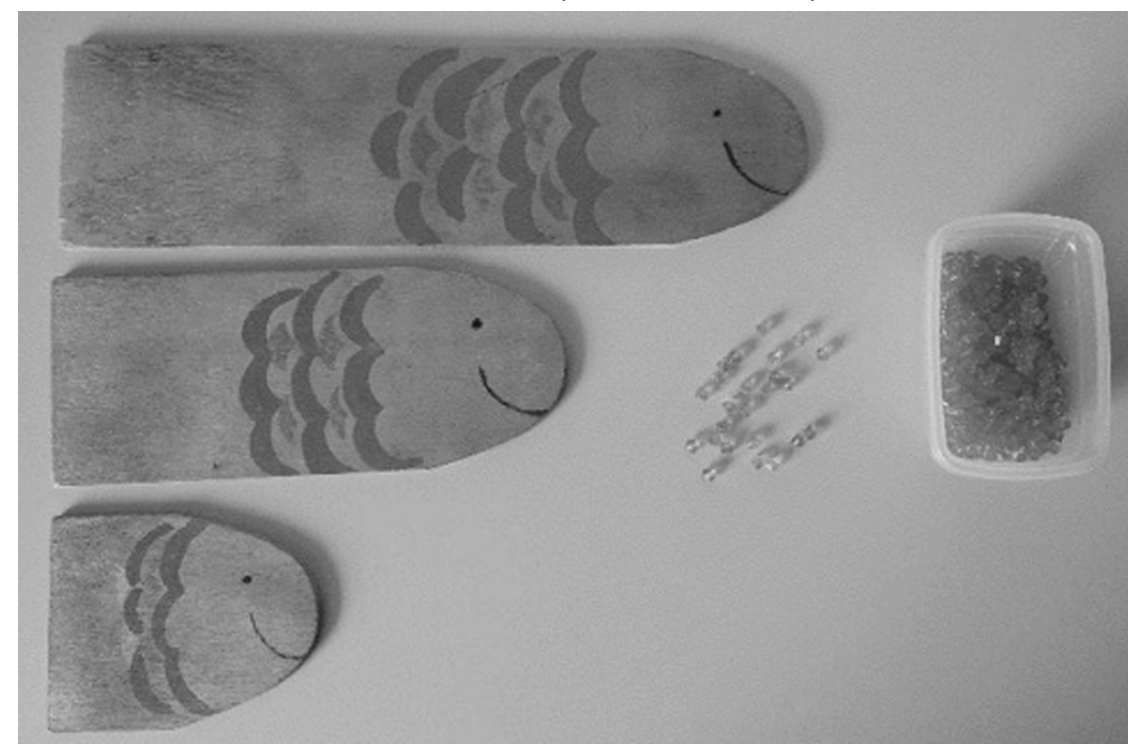

Fuente: Fotografía del material tomada por los autores 
Cada pez come de acuerdo a su tamaño, en una proporción de 1 a 3 (por ejemplo, si el pez A come 3 bolitas, el pez B come 6 bolitas y el pez C 9 bolitas). En cada pregunta de la tarea, el evaluador alimentó a uno de los peces y seguidamente el estudiante debió alimentar a los otros dos. Las instrucciones que el evaluador siguió en la aplicación de este ejercicio se presentan a continuación:

Instrucciones para la aplicación de la tarea del pensamiento multiplicativo

a) Colocar los tres peces frente al estudiante.

Figura 3- Peces para la tarea de pensamiento multiplicativo
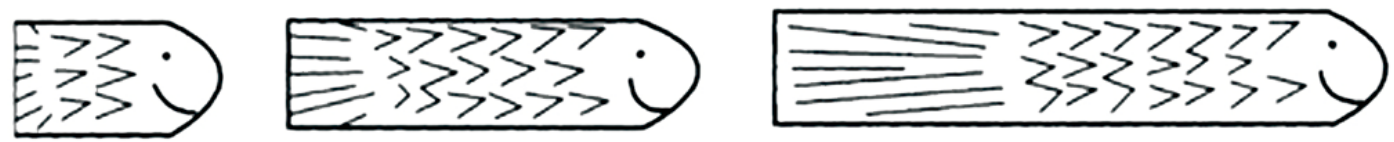

A

B

C

Fuente: Clark y Kamii, (1996, p. 42).

b) Señalando el pez B, se le dice al evaluado: "Este pez come dos veces lo que este otro pez" (señalando ahora al pez A).

c) Seguidamente, señalando el pez C, se dice: "Y este pez come tres veces lo que este otro pez" (señalando ahora al pez A).

d) Luego, con respecto al pez B se dice: "Este pez come dos veces que lo que este otro pez ( $p e z A)$, pues es dos veces más grande”. Luego de realizar esta afirmación, el evaluador deberá colocar el pez $A$ sobre el pez $B$ de tal manera que se haga notorio que el pez $B$ tiene el doble de tamaño que el pez $A$.

e) Nuevamente, el evaluador dice, refiriéndose ahora al pez C: "Este pez come tres veces lo que este otro pez (pez A), pues es tres veces más grande”. Luego de realizar esta afirmación el evaluador deberá colocar el pez $A$ sobre el pez $\mathrm{C}$ de tal manera que se haga notorio que el pez $C$ tiene el triple de tamaño que el pez $A$.

f) Para verificar que el niño ha comprendido las proporciones antes explicadas se le plantea se le dice: "Si el pez A come 3 bolitas de alimento, ¿cuántas bolitas come el pez C?"

g) Después de haber comprobado que el evaluado ha comprendido la consigna se aplicará el procedimiento con las siguientes cantidades:

- Cuando el pez $B$ recibe 4 bolitas.

- Cuando el pez $C$ recibe 9 bolitas.

- Cuando el pez $A$ recibe 4 bolitas

- Cuando el pez $A$ recibe 7 bolitas.

Siguiendo a Clark y Kamii (1996), existen cinco posibles niveles de respuesta: 
Tabla 2- Niveles de respuesta para ejercicio de pensamiento multiplicativo

Nivel

Nivel
Nivel 1: Ausencia de correspondencia serial (1a) 0
Correspondencia serial cualitativa (1b)

Nivel 2: Pensamiento aditivo con secuencia numérica.

Nivel 3: Pensamiento aditivo que implica (+2) en el
caso de B y (+3) en el caso de C Nivel 4a: Pensamiento multiplicativo sin éxito
inmediato.

Nivel 4b: Pensamiento multiplicativo con éxito inmediato.
Descripción del nivel

Las respuestas de este nivel no se encuentran seriadas según el tamaño de los peces. De encontrarse seriadas, solo se observa una correspondencia cualitativa, es decir, un orden por medio de los enunciados más o menos

Las respuestas de este nivel presentan un pensamiento aditivo que sirve para crear una secuencia utilizando el $(+1) 0$ el (+2). El evaluado solo piensa en la pareja A - B y en la pareja B - C, le resulta complicado establecer la relación $A-C$. Adicionalmente, cuando se le pregunta por $B$, opta por darle $(A+1) 0(A+2)$, si es que se le pregunta por $C$ opta por darle $(B+1) 0(B+2)$.

Las respuestas de este nivel evidencian un entendimiento superior de la consigna propuesta por el entrevistador, es decir, darle a B dos veces que a $A$ y a $C$ tres veces que a $A$. Sin embargo, al ejecutar la tarea recurren a la suma para representar ese dos veces y tres veces.

Las respuestas evidencian un pensamiento multiplicativo, sin embargo, no se encuentra del todo logrado. Los evaluados que se encuentran en este nivel de desarrollo necesitan de la estimulación y la repregunta para poder lograr respuestas acertadas.

Las respuestas evidencian haber logrado con éxito construir el pensamiento multiplicativo. Se responde a las preguntas de manera acertada y no cambian sus respuestas a pesar de ser sugestionados.

Fuente: Clark y Kamii (1996, p. 42).

\section{Resultados}

Las respuestas de los estudiantes se organizan en seis niveles de desarrollo, que corresponden a los encontrados por Clark y Kamii (1996) al realizar la misma evaluación con estudiantes de una escuela pública en Birminghan, Alabama. En la siguiente tabla se presentan los niveles de desarrollo obtenidos en cada pregunta por cada uno de los participantes.

A continuación, se describen y ejemplifican los niveles encontrados.

\section{Nivel 1a: Ausencia de correspondencia serial}

Las respuestas encontradas en este nivel no presentan una correspondencia serial. Los niños no toman en cuenta el tamaño de los peces al momento de alimentarlos; les dan más alimento a los peces más pequeños y menos alimento a los peces más grandes, 0 igualan la cantidad de alimento para peces de distinto tamaño. Este tipo de respuesta se ve en el siguiente ejemplo: 
Les (9 años): Ahora le quiero dar de comer a este pez chiquito (Pez A), le doy de comer 4 bolitas de alimento, ¿le puedes dar de comer a este otro pez (Pez B)? - (Le da dos bolitas) - ¿Y al otro pez (Pez C) cuánto le darías? - 4 bolitas. (Año 2015 - Bethel).

Tabla 3- Nivel de desarrollo obtenido en cada pregunta

\begin{tabular}{|c|c|c|c|c|}
\hline Nombre & $\begin{array}{l}\text { Prueba } 1 \\
\text { Pez B (4) }\end{array}$ & $\begin{array}{l}\text { Prueba } 2 \\
\text { Pez C (9) }\end{array}$ & $\begin{array}{l}\text { Prueba } 3 \\
\text { Pez A (4) }\end{array}$ & $\begin{array}{l}\text { Prueba } 4 \\
\text { Pez A (7) }\end{array}$ \\
\hline Wil & $1 \mathrm{a}$ & $1 \mathrm{~b}$ & $1 \mathrm{a}$ & $1 \mathrm{~b}$ \\
\hline Luc & $1 \mathrm{~b}$ & $1 \mathrm{~b}$ & 2 & 2 \\
\hline Les & $1 \mathrm{~b}$ & $1 \mathrm{~b}$ & $1 \mathrm{a}$ & $1 \mathrm{~b}$ \\
\hline Man & $1 \mathrm{~b}$ & $1 \mathrm{a}$ & $1 \mathrm{a}$ & $1 \mathrm{~b}$ \\
\hline Dal & $4 \mathrm{~b}$ & $4 \mathrm{~b}$ & $4 \mathrm{~b}$ & $4 \mathrm{~b}$ \\
\hline Sha & $4 \mathrm{a}$ & 3 & 3 & 3 \\
\hline Maz & 2 & 2 & 2 & 2 \\
\hline Yal & $1 \mathrm{~b}$ & $1 b$ & 2 & 2 \\
\hline Key & $1 \mathrm{~b}$ & $1 \mathrm{~b}$ & $1 \mathrm{~b}$ & $1 \mathrm{~b}$ \\
\hline Pat & $1 \mathrm{a}$ & $1 \mathrm{~b}$ & $1 \mathrm{~b}$ & 2 \\
\hline Ale & $1 \mathrm{~b}$ & $1 \mathrm{~b}$ & $1 \mathrm{~b}$ & $1 \mathrm{~b}$ \\
\hline Gis & $1 \mathrm{~b}$ & $1 \mathrm{~b}$ & 2 & $1 \mathrm{~b}$ \\
\hline Ste & 2 & $1 \mathrm{~b}$ & 2 & 2 \\
\hline Cri & $1 \mathrm{~b}$ & $1 \mathrm{~b}$ & $1 \mathrm{~b}$ & $1 \mathrm{~b}$ \\
\hline
\end{tabular}

Fuente: Elaborado por los autores.

Leyenda: Ausencia de correspondencia serial (Nivel 1a) - Correspondencia serial cualitativa (Nivel 1b) - Pensamiento aditivo con secuencia numérica (Nivel 2) - Pensamiento aditivo que implica +2 en el caso de B y +3 en el caso de C (Nivel 3) - Pensamiento multiplicativo son éxito inmediato (Nivel 4a) - Pensamiento multiplicativo con éxito inmediato (Nivel 4b).

Les da de comer menos alimento a los peces $\mathrm{B}$ y $\mathrm{C}$ que al pez $\mathrm{A}$, a pesar de que este último es el de menor tamaño. Luego, aun cuando el investigador le recuerda a Les la consigna de la tarea, ella entrega al pez B la misma cantidad de alimento que al pez A, y al pez $\mathrm{C}$ una cantidad inferior que a los dos anteriores:

Una pregunta, ¿cuál pez es más grande, este de acá (Pez B) o este otro de acá (Pez A)? - Este de acá (Pez C). - Claro, ese es el más grande de todos, ¿pero entre este de acá (Pez A) y este de acá (Pez B)?. - Este (Pez B). - ¿Y cuál está comiendo más ahorita? - Este pez (Pez A) - ¿Recuerdas cómo debemos darles de comer? ¿Se le da más comida al más grande o al más chiquito? - Al más grande - ¿Y cuánto le darías a este pez (Pez B) entonces? - 4 bolitas - Igual que a este pez (Pez A) entonces. ¿Y cuánto le darías a este (Pez C)? ¿Lo dejarías en 4 bolitas? ¿Cuánto le darías? - 3 bolitas. (Año 2015 - Bethel).

\section{Nivel 1b: Correspondencia serial cualitativa}


Las respuestas encontradas en este nivel mantienen una correspondencia serial, pero únicamente a un nivel cualitativo. Los estudiantes alimentan a los peces considerando, de manera general, el tamaño de los mismos: siempre un pez más grande comerá más alimento que un pez pequeño. Sin embargo, la diferencia entre la cantidad de alimento que recibe el pez B en comparación al pez A no es constante en relación a la cantidad de alimento que recibe el pez $\mathrm{C}$ en comparación al pez B, es decir, no se respeta la proporción. Este tipo de respuesta se puede evidenciar en siguiente ejemplo:

Ale (9 años): Se me ocurre que hoy día le voy a dar de comer a este pez mediano (Pez B) cuatro bolitas de alimento (coloca cuatro bolitas frente a Pez B): uno, dos, tres, cuatro. Ahora, si este pez mediano (Pez B) come cuatro bolitas de alimento, ¿cuánto crees que debería comer el más chico (Pez A)? Uno A ver, dale de comer (coloca una bolita frente a Pez A) ¿Y cuánto debería comer este pez, el más grande (Pez C)? Cinco A ver, dale de comer (coloca cinco bolitas frente a Pez C). (Año 2016 - Bena Jema).

En su respuesta, se encuentra que Ale respeta la secuencia serial cualitativa de entregar al pez A la menor cantidad de alimento, y al pez C la mayor cantidad. Sin embargo, no considera las relaciones proporcionales entre los peces (pez A requiere la mitad de alimento que $B$; pez $C$ requiere el triple de alimento que A). Así, a pesar de que se le recuerda la consigna, Ale mantiene sus respuestas ante la repregunta, aun cuando indica que el factor de decisión fue el tamaño de los peces:

A ver, entonces: yo le había dado de comer al pez mediano (Pez B) cuatro bolitas de alimento, y tú al chico le diste uno. ¿Cómo así decidiste que el pez más chico debía comer uno? ¿Cómo llegaste a esa conclusión? Porque es chico. Pero, a ver, entonces: ¿te acuerdas que este pez de acá, mediano (Pez B), es dos veces el tamaño que el chico (Pez A, que es colocado dos veces sobre Pez B)? ¿Sí? Y hemos visto que cada uno comía según su tamaño, ¿no? Entonces, si este pez (Pez B) come cuatro bolitas de alimento, ¿estás seguro que el más chico debería comer uno? (asiente con la cabeza) ¿Sí, seguro? (asiente con la cabeza) Segurísimo. ¿Y cómo llegaste a la conclusión de que el más grande debía comer cinco bolitas de alimento? Porque es el grande. (Año 2016 - Bena Jema)

\section{Nivel 2: Pensamiento aditivo con secuencia numérica}

Las respuestas en este nivel mantienen una correspondencia serial y, además, entienden la secuencia numérica entre la cantidad de alimento que recibe cada pez. Esto quiere decir que comprenden que la diferencia entre la cantidad de alimento que recibe el pez B en comparación al pez A debe ser constante en relación a la cantidad de alimento que recibe el pez $\mathrm{C}$ en comparación al pez B. Este tipo de respuesta se puede evidenciar en el siguiente ejemplo:

Man (9 años): Ahora yo le voy a dar de comer al pez más chiquito (Pez A) 7 bolitas de alimento, ¿cuántas bolitas le darías tú a este otro pez (Pez B)? -8 bolitas - ¿cuántas bolitas de alimento le 
darías a este otro pez (Pez C)? - 9 bolitas (Se le recuerdan las reglas de proporción y no modifica su respuesta). (Año 2015 - Bethel).

Así, Man cumple con mantener la correspondencia serial entre los peces, de manera que el pez $\mathrm{C}$ necesariamente recibe más alimento que $\mathrm{B}$, y este último necesariamente más que A. Sin embargo, Man no respeta las relaciones proporcionales entre los peces, sino que se limita a mantener una secuencia numérica de tipo $(+1)$.

\section{Nivel 3: Pensamiento aditivo que implica (+2) en el caso de B y (+3) en el caso de C}

Las respuestas en este nivel toman en cuenta las nociones de doble y triple. Sin embargo, al no contar aún con un pensamiento multiplicativo consolidado, traducen estas nociones a un pensamiento aditivo, entendiendo que el pez $\mathrm{B}$ debe recibir como alimento $(A+2)$ y que el Pez $C$ debe recibir como alimento $(A+3)$ o $(B+3)$. Este tipo de respuesta se puede evidenciar en el siguiente ejemplo:

Sha (11 años): Ahora yo le quiero dar 7 bolitas de alimento a este pez (Pez A) ¿cuánto le darías a este otro pez (Pez B)? - 9 bolitas de alimento. - ¿Y cuánto le darías a este otro pez (Pez C)? - 12 bolitas. (Año 2015 - Bethel).

En su respuesta, Sha otorga al pez $\mathrm{B}$ dos bolitas de alimento más que al pez $\mathrm{A}, \mathrm{y}$ al pez $\mathrm{C}$ tres bolitas de alimento más que al pez $\mathrm{B}$, coincidiendo con la descripción del nivel previamente realizada. Esta tendencia se repite también en otra de las pruebas del mismo niño:

Sha (11 años): Mira, ahora le quiero dar a este pez (Pez C) 9 bolitas de alimento, ¿cuántas bolitas de alimento le darías tú a este pez (Pez A)? - 4 bolitas de alimento. - ¿Y cuántas bolitas de alimento le darías a este otro pez (Pez B)? - 6 bolitas de alimento. (Año 2015 - Bethel).

\section{Nivel 4a: Pensamiento multiplicativo sin éxito inmediato}

En este nivel los niños evidencian ya un pensamiento multiplicativo, aunque no del todo logrado, y necesitan de la estimulación y la repregunta para poder lograr respuestas acertadas, tal como se ve en el ejemplo siguiente:

Sha (11 años): Ahora, yo quiero dar de comer a este pez del medio (Pez B) y le doy de comer 4 bolitas de alimento, ¿cuánto le darías tú a este pez (Pez A)? - (Le da dos bolitas de alimento) - ¿Y cuánto debería comer este si este (Pez C) come tres veces lo que este (Pez A)? - Cinco bolitas Entonces este (Pez A) come dos, este (Pez B) come 4 y este (Pez C) come 5 - Sí - ¿Y estaría bien si yo le doy de comer a este (Pez B) cinco bolitas y a este (Pez C) 4 bolitas? - No - ¿Y si le doy de comer 4 bolitas a este (Pez A) y 2 bolitas a este (Pez B)? ¿Estaría bien? - No - ¿Y qué tal si le doy de comer a este (Pez C) 8 y a este (Pez B) lo dejo en 4 bolitas? ¿Estaría bien? - No - Ahora, ¿recuerdas que este pez (Pez C) debe comer tres veces lo que come este pez (Pez A)? -Si - ¿cuánto 
come este pez (Pez A)? - 2 bolitas - ¿Entonces cuánto debe comer este pez (Pez C)? - 6 bolitas. (Año 2015 - Bethel).

Se observa que, en todo momento, Sha mantiene la correspondencia serial cualitativa entre los peces: así, a pesar de la contra-sugestión donde el evaluador trata de invertir las cantidades que les son dadas a los peces A y B, Sha mantiene su respuesta. Asimismo, logra desde el principio dar al pez A la cantidad de bolitas que le corresponde (dos bolitas), aunque se equivoca en el caso del pez C (le da cinco bolitas). Sin embargo, es capaz de corregirse luego de que el investigador le recuerda la consigna de que el pez $\mathrm{C}$ come tres veces lo que come el pez A.

\section{Nivel 4b: Pensamiento multiplicativo con éxito inmediato}

Los niños que se encuentran en este nivel han logrado con éxito construir el pensamiento multiplicativo. Responden a las preguntas de manera acertada y no cambian sus respuestas a pesar de ser sugestionados. A continuación, presentamos algunos ejemplos:

Dal (11 años): Supongamos que yo le quiero dar de comer a este pez de acá (Pez B) y le doy cuatro bolitas de alimento, ¿cuánto le darías de comer tú a este (Pez A)? - Dos bolitas - ¿Y a este? -6 bolitas - Y, por ejemplo, ¿Estaría bien si en vez de darle 6 bolitas a este pez (Pez C) le damos 5? - No - ¿cuánto tenemos que darle? - 6 bolitas - ¿Y si le damos 7 bolitas? ¿Estaría bien? -No. (Año 2015 - Bethel).

Como se puede observar en el problema anterior, Dal comprende claramente la consigna desde el primer momento, y es capaz de entregar a cada pez la cantidad de alimento que le corresponde siguiendo las proporciones del tamaño de cada uno. Sin embargo, se puede notar también que aunque se reafirma en su respuesta luego de cada repregunta, presenta dificultades para explicar las razones de la misma.

Dal (11 años): Ahora le quiero dar de comer a este pez (Pez A) 4 bolitas de alimento, ¿cuánto le darías de comer a este pez (Pez B)? - (Le da de comer 8 bolitas) - ¿Y cuánto le darías de comer a este otro pez (Pez C)? - 12 bolitas - ¿cómo sabes cuánta comida darle a cada uno? si te encontraras con un niño pequeño que no sabe darles de comer, ¿cómo le explicarías? - Le diría que le dé así, 4 bolitas a este (Pez A), 8 bolitas a este (Pez B) y 12 bolitas a este (Pez C). (Año 2015 - Bethel).

\section{Conclusiones y discusión}

El presente estudio tuvo como objetivo indagar el razonamiento multiplicativo de un grupo de niños pertenecientes a dos comunidades shipibo-konibo de la región Ucayali del Perú, mediante el método clínico-crítico de Jean Piaget (DELVAL, 2001; 
Jorge Villalba GARCÉS; Susana Frisancho HIDALGO

DUCRET, 2004; PARRAT, 2016) utilizando una tarea de multiplicación propuesta por Clark y Kamii (1996).

Las principales conclusiones del estudio son las siguientes:

a) El pensamiento multiplicativo de este grupo de niños shipibo-konibo sigue el mismo patrón de desarrollo mostrado en estudios clásicos sobre este tema, realizados con niños occidentales.

b) En general, los niños mostraron dificultades en la operación de multiplicación.

c) El desarrollo del pensamiento multiplicativo de los niños que participaron de este estudio toma más tiempo, comparativamente, al que toma en niños no indígenas; en este grupo de niños shipibo-konibo los niveles de desarrollo esperados se alcanzan después.

Los resultados han permitido observar una línea de desarrollo del pensamiento multiplicativo coherente con estudios realizados previamente en contextos culturales muy distintos (CLARK; KAMII, 1996; PIAGET, 1975). De este modo, este estudio puede considerarse una prueba más de la universalidad de las estructuras que sostienen el conocimiento lógico-matemático (PIAGET, 1971), la que resulta posible porque los seres humanos, más allá de las particularidades de la cultura en la que se encuentran, cuentan con el mismo aparato psíquico para asimilar y acomodar la información de su entorno y pueden construir, en consecuencia, las mismas estructuras lógico-matemáticas para organizarlo (HALLPIKE, 1986; PIAGET, 1977).

La investigación ha mostrado las dificultades que tienen estos niños, a distintas edades, para comprender y ejecutar la operación de multiplicación, aun cuando se usó material manipulativo que facilitó la tarea y permitió a los niños tener un mejor desempeño. Como ya hemos señalado, en el Perú los resultados de las evaluaciones nacionales de rendimiento en matemáticas muestran los problemas que tienen los estudiantes de educación básica en esta disciplina. En el currículo nacional peruano, la multiplicación se presenta como meta pedagógica ya en el segundo grado de primaria; en este grado se espera que el estudiante resuelva los problemas con sumas repetidas, mientras que en tercer grado se plantea que ya multiplique números de una y dos cifras. Sin embargo, la literatura especializada sugiere que, si bien el pensamiento multiplicativo aparece ya en algunos niños de segundo grado, su desarrollo es extremadamente lento, por lo que estas metas resultan demasiado elevadas para los primeros años de primaria (CLARK; KAMII, 1996). Así, el bajo rendimiento de los estudiantes en matemáticas puede explicarse, en cierta medida, por esta inadecuación curricular.

La memorización de las tablas de multiplicar, que es un método de enseñanza frecuente en las escuelas, resulta una mala estrategia que no solo es complicada para los niños, sino que puede perjudicar el desarrollo de las nociones que permiten comprender esta operación, además de anular la iniciativa de los estudiantes para realizar el ejercicio mental de operar de manera multiplicativa (DUCKWORTH, 1987). En efecto, si bien el uso 
de algoritmos ayuda a agilizar los cálculos, su uso precoz puede interferir con el desarrollo del pensamiento multiplicativo (SILVA et al., 2015) o ayudar a calcular más rápidamente solo cuando el operador es un cierto número, por ejemplo, el 5 (CAMPBELL; GRAHAM, 1985; LEFEVRE et al., 1996). Enseñar la multiplicación solo como una suma abreviada también produce problemas y puede interferir posteriormente con el desarrollo de nociones tales como razones y proporciones o relaciones algebraicas (HURST; HURRELL, 2014; FERNANDEZ; LLINARES, 2010). Como afırman Clark y Kamii (1996), los niños no deben ser forzados a aprender algoritmos de cálculo y estrategias que no pueden aun hacer ni comprender; diferentes niños muestran diferentes modos de abordar la multiplicación, lo que es una fuente de información importante para el docente acerca de cómo conceptualizan la operación (MABBOTT; BISANZ, 2003; STANIC; KILLION; STEFFE, 1989).

Finalmente, es relevante destacar que los niveles de desarrollo del pensamiento multiplicativo de los niños participantes de este estudio se están logrando tardiamente, en comparación con los que muestran estudios realizados con otras poblaciones. Si bien la teoría piagetiana presenta los niveles de desarrollo de cualquier noción indicando edades aproximadas para el paso de un estadio al otro, afırma también que la velocidad de desarrollo de las nociones depende mucho de la estimulación del mundo exterior y de los intercambios que el niño tenga con él (DONG0, 2002; HALLPIKE, 1986; PIAGET, 1977). En este sentido, si bien los objetos que forman parte de los problemas de multiplicación en una comunidad shipibo-konibo pueden ser distintos que los objetos que forman parte de un ejercicio de multiplicación en una escuela de Lima, la actividad multiplicativa que se realiza con ellos y que da forma a las estructuras de pensamiento es universal y sigue siempre una misma secuencia (PIAGET; SZEMINSKA, 1975). Como sabemos que los estudiantes logran mejores resultados de aprendizaje cuando la enseñanza se relaciona con el mundo real y está contextualizado culturalmente, y que estos resultados abarcan diferentes aspectos de su desarrollo tales como el desempeño académico, el interés en la materia o el fortalecimiento de la identidad cultural (DEWAH; VAN WYK, 2014; NKHWALUME; LIU, 2013; TILLEMA, 2012; NUNES, 1993; NUNES; CARRAHER; DIAS SCHLIEMANN, 1982; 1993; NUNES; BRYANT, 1996; NAM; ROEHRIG; KERN; REYNOLDS, 2013), resultaría entonces muy importante incorporar en las actividades escolares actividades culturales de la vida cotidiana ligadas a la multiplicación, y proveer al estudiante de situaciones matemáticas significativas, a fin de consolidar de mejor modo esta operación y ayudar a su desarrollo.

\section{Referencias}

AROCA, Armando. El programa etnomatemática: avances, desafíos y su papel en la globalización económica y el proyecto neoliberal. Revista Latinoamericana de Etnomatemática, San Juan de Pasto, v. 9, n. 2, p. 238277, jun. 2016.

ASCHER, Marcia. Mathematics elsewhere: an exploration of ideas across cultures. New Jersey: Princeton University Press, 2002.

BESSA, Sonia; GONÇALVES DA COSTA, Váldina. Jogo sempre 12: opção à compreensão das operações aritméticas. Schème, Marília, v. 8, n. 1, p. 36-65, 2016. 
BISHOP, Alan. Western mathematics: the secret weapon of cultural imperialism. In: ASHCROFT, Bill; GRIFFITHS, Gareth; TIFFIN, Helen (Ed.). The post-colonial studies reader. London: Routledge, 1995. p. 71-76.

BRUNER, Jerome; OLVER, Rose; GREENFIELD, Susan. Sobre la conservación de los líquidos. In: BRUNNER, Jerome; OLVER, Rose; GREENFIELD, Susan. Studies in cognitive growth. New York: Wiley, 1966. p. 183-197.

CAMPBELL, Jaimie; GRAHAM, David. Mental multiplication skill: Structure, process, and acquisition. Canadian Journal of Psychology, Ontário, v. 39, n. 2, p. 338-36, 1985.

CARRAHER, Terezinha; CARRAHER, David; SCHILEMANN, Analucía. En la vida diez, en la escuela cero. México, DC: Siglo Veintiuno, 1991.

CARRETERO, Mario. La teoría de Piaget y la psicología transcultural: la búsqueda de los universales cognitivos. Infancia y Aprendizaje, Madrid, v. 4, n. 2, p. 187-199, 1981.

CLARK, Faye; KAMII, Constance. Identification of multiplicative thinking in children in grades 1-5. Journal for Research in Mathematics Education, Delaware, v. 27, n. 1, p. 41-51, 1996.

COSTA, Lucélida da; LUCENA, Isabel. Educação matemática na escola indígena: implicações à formação de professores. Unión, Madrid, v. 44, p. 73-89, 2015.

COX, Linda. Analysis, classification, and frequency of systematic error computational patterns in the addition, subtraction: multiplication, and division vertical algorithms for grades 2-6 and special education classes. ED 092 407. Kansas: Kansas University, 1974.

D'AMBROSI0, Ubiratan. Ethnomathematics and its place in the history and pedagogy of mathematics. For the Learning of Mathematics, Ottawa, v. 5, n. 1, p. 44-48, 1985.

D’AMBROSI0, Ubiratan. Las bases conceptuales del programa etnomatemática. Revista Latinoamericana de Etnomatemática, San Juan de Pasto, v. 7, n. 2, p. 100-107, 2014.

D'AMBROSI0, Ubiratan. What is ethnomathematics and how can it help children in schools? Teaching Children Mathematics, Nebraska, v. 7, n. 6, p. 308-310, 2001.

DELVAL, Juan. Descubrir el pensamiento de los niños: introducción a la práctica del método clínico-crítico. Barcelona: Paidós, 2001.

DEWAH, Charity; VAN WYK, Micheal. The place of indigenous cultural games by educators in the teaching and learning of mathematics. Journal of Human Ecology, New Delhi, v. 48, n. 1, p. 189-197, 2014.

DONGO, Adrián. Piaget y los niños marginados: epistemología genética, diagnóstico y soluciones. Lima: Pakyu, 2002.

DUCKWORTH, Eleanor. Some Depths and perplexities of elementary arithmetic. Journal of Mathematical Behavior, v. 6, n. 1, p. 43-94, 1987. 
DUCRET, Jean-Jacques. Methode clinique-critique piagetienne. Genève: Service de la Recherché en Education, 2004.

FERNANDEZ, Ceneida; LLINARES, Salvador. Relaciones entre el pensamiento aditivo y mutiplicativo en estudiantes de educación primaria: el caso de la construcción de la idea de razón. Horizontes Educacionales, Chillán, v. 15, n. 1, p. 11-22, 2010.

FUENTES LEAL, Camilo. Descolonizando la escuela: ¿Es posible llevar la etnomatemática al aula? Revista Latinoamericana de Etnomatemática, San Juan de Pasto, v. 7, n. 2, p. 222-244, 2014.

GREENFIELD, Patricia; CHILDS, Carla. Weaving, color terms, and pattern representation: cultural influences and cognitive development among the Zinacantecos of Southern Mexico. International Journal of Psychology, v. 11, p. 23-48, 1977.

GREENFIELD, Patricia; MAYNARD, Ashley; CHILDS, Carla. Historical change, cultural learning, and cognitive representation in Zinacantec Maya children. Cognitive Development, v. 18, p. 455-487, 2003.

HALLPIKE, Christopher. Fundamentos del pensamiento primitivo. México, DC: Fondo de Cultura Económica, 1986.

HORSTHEMKE, Kai; SCHAFER, Marc. Does 'African mathematics' facilitate access to mathematics? Towards an ongoing critical analysis of ethnomathematics in a South African context. Pythagoras, Durbanville, v. 65, p. 2-9, 2007.

HURST, Chris; HURRELL, Derek. Developing the big ideas of number. International Journal of Educational Studies in Mathematics, Notre Dame, v. 1, n. 2, p. 1-18, 2014.

JARAMILLO, Diana. La educación matemática en una perspectiva sociocultural: tensiones, utopías, futuros posibles. Revista Educación y Pedagogía, Medellín, v. 23, n. 59, p. 13-36, 2011.

JOHN, Martha et al. Children's thinking in botswana: Piaget tasks examined. International Journal of Psychology, v. 18, n. 1-4, p. 229-238, 1983.

KAMII, Constance. Reinventando la aritmética III: implicaciones de la teoría de Jean Piaget. Madrid: Visor, 1994.

LAWRENCE, Shirley. Otthava: making baskets and doing geometry in the Makhuwa culture in the Northeast of Mozambique, by Paulus Gerdes. Journal of Mathematics and the Arts, Kansas, v. 5, n. 2, p. 101-102, 2011.

LEFEVRE, Jo-Anne et al. Multiple routes to solution of single-digit multiplication problems. Journal of Experimental Psychology, Missouri, v. 125, n. 3, p. 284-306, 1996.

MABBOTT, Donald; BISANZ, Jeffrey. Developmental change and individual differences in children's multiplication. Child Development, Chicago, v. 74, n. 4, p. 1091-1107, 2003. 
MEANEY, Tamsin. Symbiosis or cultural clash? Indigenous students learning mathematics. Journal of Intercultural Studies, Victoria, v. 23, n. 2, 167-187, 2002.

MEANEY, Tamsin; EVANS, Deb. What is the responsibility of mathematics education to the Indigenous students that it serves? Educational Studies in Mathematics, Netherlands, v. 82, p. 481-496, 2013.

MEANEY, Tamsin; MCMURCHY-PILKINGTON, Colleen; TRINICK, Tony. Indigenous students and the learning of mathematics. In: PERRY, Bob et al. (Ed.). MERGA four-yearly review of mathematics education research, 2008-2011. Rotterdam: Sense, 2012. p. 67-88.

MISHRA, Ramesh. Piagetian studies of cognitive development in India. Psychological Studies, New York, v. 59, p. 207-22, 2014.

MORIMAZATO IZU, Regina. Niños de la Amazonía: una experiencia de trabajo conjunto por una mejor educación para los niños y las niñas asháninkas de la selva central del Perú. Educación, Lima, v. 20, n. 39, p. 73-92, 2011. (10199403).

NAM, Younkyeong; ROEHRIG, Gillian; KERN, Anne Liu. \& REYNOLDS, Bree. Perceptions and practices of culturally relevant science teaching in American Indian classrooms. International Journal of Science \& Mathematics Education, New York, v. 11, n. 1, 143-167, 2013.

NKHWALUME, Liu. Using technology to teach mathematical concepts through cultural designs and natural phenomena. Asian Journal of Management Sciences and Education, Chikusei-shi, v. 2, n. 2, 25-35, 2013.

NUNES, Terezinha. The socio-cultural context of mathematical thinking: research findings and educational implications. In: BISHOP, Alan et al. (Ed.). Significant influences on children's learning of mathematics. Paris: Unesco, 1993. p. 27-42.

NUNES, Terezinha; BRYANT, Peter. Children doing mathematics. Oxford: Blackwell, 1996.

NUNES, Terezinha; CARRAHER, David; DIAS SCHLIEMANN, Analucía. Na vida, dez; na escola, zero: os contextos culturais da aprendizagem da matemática. Cadernos de Pesquisa, São Paulo, n. 42, p. 79-86, 1982.

NUNES, Terezinha; CARRAHER, David; DIAS SCHLIEMANN, Andalucía. Street mathematics and school mathematics. New York: Cambridge University Press, 1993.

NYITI, Raphael. The development of conservation in the meru children of Tanzania. Child Development, Michigan, v. 47, n. 4, p. 1122-1129, 1976.

PAIS, Alexandre. Criticisms and contradictions of ethnomathematics. Educational Studies in Mathematics, Heidelberg, v. 76, n. 2, p. 209-230, 2011.

PARRA, Aldo; ORJUELA, Jorge. Consideraciones sobre educación matemática y educación indígena en Colombia. Revista Latinoamericana de Etnomatemática, San Juan de Pasto, v. 7, n. 2, p. 181-201, 2014. 
PARRAT, Silvia. Conversaciones libres con los niños: el método clínico Piagetiano. Relación entre teoría y método. In: FRISANCHO, Susana (Coord.). Ensayos constructivistas. Lima: PUCP, 2016. p. 51-76.

PERÚ. Ministerio de Cultura. Base de datos de pueblos indígenas u originarios. 2017. Disponible en: <http:// bdpi.cultura.gob.pe/lista-de-pueblos-indigenas>. Acceso en: 5 jul. 2016.

PERÚ. Ministerio de Educación. Programa curricular de educación primaria. Lima: Minedu, 2016.

PIAGET, Jean. Introducción a la epistemología genética: el pensamiento matemático. Buenos Aires: Paidós, 1975.

PIAGET, Jean. Need and significance of cross-cultural studies genetic psychology. In: INHELDER; Bärbel.; CHIPMAN, Harold; ZWINGMANN, Charles (Ed.). Piaget and his school: a reader in developmental psychology. New York: Springer Science \& Business Media, 1974. p. 259-268.

PIAGET, Jean. Psicología y epistemología. Barcelona: Ariel, 1971.

PIAGET, Jean. Seis estudios de psicología. Barcelona: Seix Barral, 1977.

PIAGET, Jean; INHELDER, Bärbel. Psicología del niño. Madrid: Morata, 2007.

PIAGET, Jean; SZEMINSKA, Alina. Génesis del número en el niño. Buenos Aires: Guadalupe, 1975.

RITCHIE, Stuart; BATES, Timothy. Enduring links from childhood mathematics and reading achievement to adult socioeconomic status. Psychological Science, Victoria, v. 24, n. 7, p. 1301-1308, 2013.

ROWLANDS, Stuart; CARSON, Robert. Where would formal, academic mathematics stand in a curriculum informed by ethnomathematics? A critical review of ethnomathematics. Educational Studies in Mathematics, Netherlands, v. 50, p. 79-102, 2002.

SANTOS, Lucí; DONIZETI, Ademir. Educação escolar indígena, matemática e cultura: a abordagem etnomatemática. Revista Latinoamericana de Etnomatemática, San Juan de Pasto, v. 4, n. 1, p. 21-39, 2011.

SILVA, João da et al. Estratégias e procedimentos de crianças do cliclo de alfabetização frente à situações-problema que envolvem multiplicação e divisão. Educação Matemática Pesquisa, São Paulo, v. 17, n. 4, p. 740-766, 2015.

SCHLIEMANN, Analucía et al. Use of multiplicative commutativity by school children and street sellers. Journal for Research in Mathematics Education, Delaware, v. 29, n. 4, p. 422-435, 1998.

SCHROEDER, Joachim. Más allá de los platos típicos: el proyecto matemática intercultural en el Perú. Cuadernos Intercultuarles, Valparaíso, v. 3, n. 4, p. 51-63, 2005.

SIEGLER, Robert; BRAITHWAITE, David. Numerical development. Annual Review of Psychology, New Jersey, v. 68, p. $187-213,2017$. 
SIEMON, Dianne; BLEACKLY, John; NEAL, Denise. Working with the big ideas in number and the Australian curriculum: mathematics. In: ATWEH, Bill et al. (Ed.). Engaging the Australian national curriculum mathematics: perspectives from the field. Otawa: Mathematics Education Research Group of Australasia, 2012. p. 19-45.

SIEMON, Dianne et al. Scaffolding numeracy in the middle years-project findings, materials, and resources. Final Report Retrieved, n. 27, p. 08-16, 2006.

SKOVSMOSE, Ole. Linking mathematics education and democracy: citizenship, mathematical archaeology, mathemacy and deliberative interaction. Zentralblatt füur Didaktik der Mathematik, Berlín, v. 30, n. 6, p. 195-203, 1998. Disponible en: <https://doi.org/10.1007/s11858-998-0010-6>. Acceso en: 5 jul. 2016.

STANIC, George; KILLION, Kurt; STEFFE, Leslie. Children's multiplication. The Arithmetic Teacher, v. 37, n. 1, p. 34-36, 1989.

SUBRAMANIAN, Jayasree. One mathematics for all: can it be realized in a multicultural, multilingual country? Intercultural Education, London, v. 26, n. 4, p. 266-277, 2015.

TILLEMA, Erik. What is the difference? Using contextualized problems. Mathematics Teaching in the Middle School, Virginia, v. 17, n. 8, 472-478, 2012.

UMC. Unidad de Medición de Calidad Educativa. Resultados de la evaluación censal de estudiantes 2013. Lima: UMC, 2013. Disponible en: <http://umc.minedu.gob.pe/evaluacion-censal-de-estudiantes-2013ece-2013/>. Acceso en: Acceso en: 5 jul. 2016.

UMC. Unidad de Medición de Calidad Educativa. Resultados de la evaluación censal de estudiantes 2014. Lima: UMC, 2014. Disponible en: <http://umc.minedu.gob.pe/evaluacion-censal-de-estudiantes-2014ece-2014/>. Acceso en: Acceso en: 5 jul. 2016.

UMC. Unidad de Medición de Calidad Educativa. Resultados de la evaluación censal de estudiantes 2015. Lima: UMC, 2015. Disponible en: <http://umc.minedu.gob.pe/evaluacion-censal-de-estudiantesece-2015/>. Acceso en: Acceso en: Acceso en: 5 jul. 2016.

UMC. Unidad de Medición de Calidad Educativa. Resultados de la evaluación censal de estudiantes 2016. Lima: UMC, 2016. Disponible en: <http://umc.minedu.gob.pe/resultadosece2016>. Acceso en: Acceso en: 5 jul. 2016.

VAN DER VEN, Sanne et al. Learning multiplication: an integrated analysis of the multiplication ability of primary school children and the difficulty of single digit and multidigit multiplication problems. Learning \& Individual Differences, Houston, v. 43, p. 48-62, 2015.

VERGARA-LOPE TRISTÁN, Samana; HEVIA, Felipe; RABAY, Víctor. Evaluación ciudadana de competencias básicas de lectura y aritmética y análisis de factores asociados en Yucatán, México. Revista Iberoamericana de Evaluación Educativa, Madrid, v. 10, n. 2, p. 85-109, 2017. 
VILLALBA, Jorge; FRISANCHO, Susana. Evaluación de operaciones lógico-matemáticas mediante dos métodos distintos en niños del pueblo indígena shipibo-konibo. Revista Interdisciplinaria, Buenos Aires, v. 35, n. 1, p. 217-238, 2018.

VON RENESSE, Christine; EKCE, Volker. Mathematics and salsa dancing. Journal of Mathematics and the Arts, Kansas, v. 5, n. 1, p. 17-28, 2011.

WARES, Arsalan. Appreciation of mathematics through origami. International Journal of Mathematical Education in Science and Technology, London, v. 44, n. 2, p. 277-283, 2012.

WARES, Arsalan. Geometry embedded in North American quilt patterns, International Journal of Mathematical Education in Science and Technology, London, v. 37, n. 5, 608-613, 2006.

WARES, Arsalan. Mathematical thinking and origami. International Journal of Mathematical Education in Science and Technology, London, v. 47, n. 1, p. 155-163, 2016.

WARES, Arsalan. Using origami boxes to explore concepts of geometry and calculus. International Journal of Mathematical Education in Science and Technology, London, v. 42, n. 2, 264-272, 2010.

WARES, Arsalan; ELSTAK, Iwan. Geometry unfolding. International Journal of Mathematical Education in Science and Technology, London, v. 45, n. 4, p. 589-595, 2013.

WARES, Arsalan; ELSTAK, Iwan. Origami, geometry and art. International Journal of Mathematical Education in Science and Technology, London, v. 48, n. 2, p. 317-324, 2017.

W00DROW, Derek. Democratic Education: does it exist - especially for mathematics education? For the Learning of Mathematics, Ottawa, v. 17, n. 3, p. 11-16, 1997.

WORTHINGTON, Maulfry; VAN OERS, Bert. Pretend play and the cultural foundations of mathematics. European Early Childhood Education Research Journal, London, v. 24, n. 1, p. 51-66, 2016.

Recibido en: 23.05.2018 Revisiones en: 21.08.2018 Aprobado en: 18.02.2019

Jorge Villalba Garcés es licenciado en Psicología Educacional. Miembro del Grupo de Investigación en Cognición, Aprendizaje y Desarrollo del Departamento de Psicología y predocente de diversos cursos de la Facultad de Psicología de la Pontificia Universidad Católica del Perú.

Susana Frisancho Hidalgo es doctora en Psicología del Desarrollo, magíster en Psicología y licenciada en Psicología Educacional. Profesora principal del Departamento de Psicología y coordinadora del Grupo de Investigación en Cognición, Aprendizaje y Desarrollo del Departamento de Psicología de la Pontificia Universidad Católica del Perú. 